\title{
Perioperative chemotherapy in the management of high risk upper tract urothelial cancers
}

\author{
Matthew G. Kaag \\ Division of Urology, Department of Surgery, The Pennsylvania State College of Medicine, Hershey, PA, USA \\ Correspondence to: Matthew G. Kaag, MD. The Pennsylvania State University, College of Medicine, Division of Urology, 500 University Drive, H055, \\ Hershey, PA 17033-0850, USA. Email: mkaag@pennstatehealth.psu.edu.
}

\begin{abstract}
Radical nephroureterectomy (RNU) remains the gold-standard in the treatment of invasive urothelial cancers of the upper tract ( $>$ p T2). However, there are stage-related, postoperative recurrence and cancer-specific death rates that are unacceptably high. Multimodality treatment regimens including neoadjuvant and adjuvant cisplatin-based systemic chemotherapy have been studied. While there is a paucity of Level 1 evidence to support either regimen, both have advantages and disadvantages. The provision of chemotherapy in the neoadjuvant setting is supported by extensive bladder cancer literature, but randomized controlled trials in the upper tract have not been completed. Neoadjuvant chemotherapy also risks overtreatment of patients due to the lack of accurate pre-operative staging modalities. On the other hand, adjuvant chemotherapy is supported by the findings of one prospective randomized trial, and eliminates the need for patient selection based on imperfect pre-operative modalities. However, the rigors of surgery and the renal function loss related to nephrectomy, may preclude the provision of adjuvant chemotherapy in a significant subset of patients. One may conclude that multimodal therapy is desirable for oncologic control, but the best means of providing such therapy requires further study.
\end{abstract}

Keywords: Chemotherapy; urothelial carcinoma

Submitted Dec 31, 2019. Accepted for publication Jan 15, 2020.

doi: $10.21037 /$ tau.2020.03.48

View this article at: http://dx.doi.org/10.21037/tau.2020.03.48

\section{Introduction}

Upper tract urothelial cancer (UTUC) is a rare malignancy arising from the epithelial lining of the renal pelvis and ureters. While there are 60,000 new cases of urothelial cancer in the United states each year, only $5 \%$ of these arise in the upper tracts, giving UTUC an incidence of 1.8 cases per 100,000 person-years (1-3). UTUC afflicts primarily elderly men. The median age at diagnosis is approximately 70 , and the male to female ratio is 2:1 (2).

Most patients initially present with either gross or microscopic hematuria, which prompts a workup appropriately consisting of cystoscopy, retrograde pyelography and/or CT urography, and if indicated, ureteroscopic biopsy and/ or selective upper tract cytology (4). Rarely, UTUC may be diagnosed due to flank pain, palpable mass, or as an incidental finding on imaging.
While much more rare than urothelial cancer of the bladder, UTUC tends to present at a more advanced stage and progresses more rapidly than bladder cancer. Organ sparing local management is complicated by the difficulties of accessing the upper tract for endoscopic resection and intraluminal delivery of topical therapy. Nevertheless, nephron sparing modalities are becoming more widely accepted. Non-invasive disease, in particular low-grade, low volume disease is more frequently managed via ureteroscopic or percutaneous resection, with or without instillation of topical agents like BCG. However, the gold standard in the management of high-grade, bulky, or invasive disease remains radical nephroureterectomy (RNU) with lymph node dissection (4).

Despite aggressive surgical intervention, many patients still experience disease recurrence, and prognosis for these patients is poor. In one study $80 \%$ of patients who 
developed recurrence of their UTUC after RNU were dead of disease at 2 years (5). Patients with locally or regionally advanced disease ( $\geq \mathrm{T} 3$ and/or positive lymph nodes) at RNU are known to have poor prognosis, and for two decades outcomes in this patient population remained fairly static (6-8).

The frequency of disease recurrence after RNU and the poor outcomes experienced by these patients have prompted the adoption of multimodal therapy in the management of select high-risk UTUC patients. Over the last decade there have been multiple studies addressing the timing and tolerability of chemotherapy in the perioperative setting. While there are known barriers associated with both neoadjuvant and adjuvant chemotherapy around the time of $\mathrm{RNU}$, and a unanimous consensus on the role and timing of perioperative chemotherapy has not been reached, several published guidelines now discuss its consideration $(9,10)$.

This manuscript will examine the data addressing the expanded incorporation of perioperative chemotherapy (NAC) in the treatment of high risk UTUC, as well as the barriers to its utilization. The rationale for and tolerability of neoadjuvant and adjuvant chemotherapy will be discussed.

\section{Current guideline recommendations}

There are current guidelines addressing the management of UTUC published by the European Association of Urology (EAU) and the National Comprehensive Cancer Network (NCCN). In recent updates both organizations have begun to include suggestions regarding the role of NAC in the management of UTUC. The EAU guidelines on UTUC states that the efficacy of platinum-based chemotherapy is expected to mirror that seen in bladder cancer, but cautions that the risk of diminished glomerular filtration rate (GFR) after RNU may limit the patient's ability to receive chemotherapy (10). NAC is considered a safe option in select patients. The 2019 NCCN Guidelines on bladder cancer similarly state: "Neoadjuvant chemotherapy may be considered for select patients with UTUC, particularly for higher stage and/or grade tumors, as renal function will decline after nephroureterectomy and may preclude adjuvant therapy" (9). The 2019 NCCN management flowcharts for renal pelvic tumors and ureteral tumors both mention NAC as therapy to be 'considered' in the treatment of high grade UTUC in select patients.

\section{The natural history of UTUC}

While RNU is the gold standard therapy for high grade or large volume UTUC, survival rates following surgery remain consistently disappointing. Published survival data from the end of the last decade demonstrates 5 -year diseasespecific survival ranging from $61-76 \%(8,11)$. Cancer specific mortality is closely associated with advanced stage at the time of surgery. In one study evaluating 1363 patients undergoing RNU at twelve different institutions, greater than $50 \%$ of patients were pT2 or higher at RNU. The 5 -year disease-specific survival rates for patients with pT2, pT3, and pT4 were $75 \%, 54 \%$ and $12 \%$ respectively. $23 \%$ of patients undergoing lymph node dissection were $\mathrm{pN}+$, with an associated 5-year disease specific survival of $35 \%$. This mirrors data from 1998 when the UT Southwestern group reported a $30 \% 5$-year disease-specific survival rate for their cohort of high risk $(\geq \mathrm{pT} 3$ and/or $\mathrm{N}+\mathrm{M}+)$ patients (12). The importance of advanced disease at the time of RNU cannot be overemphasized.

More contemporary data collected on 414 patients undergoing RNU without NAC at seven academic medical centers between 2003 and 2012 revealed that $55 \%$ of patients were stage $\geq \mathrm{pT} 2,6 \%$ had a positive soft tissue margin, and of the 249 patients undergoing pathologic evaluation of lymph nodes, $11 \%$ had nodal disease (13). Adjuvant chemotherapy was used in the post-operative management of $31 \%$ of the patients in this cohort, and a small but statistically significant 5 -year cancer-specific survival benefit was seen in those patients receiving chemotherapy (39\% vs. $32 \%, \mathrm{P}=0.04$ ). However, the 5 -year cancer-specific survival for the total cohort remained only $34 \%$.

In summary, pathology reports following RNU for UTUC are likely to report $\geq \mathrm{pT} 2$ or $\mathrm{N}+$ in over half of patients, putting a significant portion of the population at high risk of recurrence. As recently as 2012, Rink et al. reported that $80 \%$ of patients experiencing recurrence after RNU will die of their cancer within 2 years (5). Thus, despite aggressive surgical management our ability to achieve acceptable cancer-specific survival for these patients is limited with a single modality approach, providing an impetus for clinicians to evaluate ways in which we might deliver multimodal therapy to the majority of patients with high risk UTUC.

\section{Neoadjuvant chemotherapy in muscle invasive bladder cancer}

Initial recommendations to include NAC in the treatment of UTUC were almost exclusively based on literature demonstrating the efficacy of platinum-based NAC in 
patients with muscle invasive bladder cancer. UTUC is a rare malignancy, and historically multimodal treatment selection has been hindered by shortcomings in staging which complicate patient selection for aggressive therapy. With minimal data specifically in the upper tract realm, the clinical community has extrapolated the findings in the bladder cancer literature to guide the utilization of perioperative chemotherapy in the upper tract.

NAC has proven efficacy in muscle invasive bladder cancer and has become, in combination with radical cystectomy and pelvic lymph node dissection, part of the standard of care. In 2003 the Southwest Oncology Group published their randomized controlled trial of radical cystectomy with or without cisplatin-based pre-operative chemotherapy (methotrexate, vinblastine, adriamycin, and cisplatin: MVAC) (14). Data from this trial demonstrated a $14 \%$ increase in disease-free 5-year survival with improved median survival (77 months NAC plus cystectomy $v s$. 46 months cystectomy alone). The evidence from other early NAC trials in MIBC was somewhat inconsistent, but in 2005 the Advanced Bladder Cancer Meta-analysis Collaboration published updated data on 11 trials encompassing 3,005 patients (15). They demonstrated a 5\% improvement in overall survival and a $9 \%$ improvement in disease-free survival at 5 years in patients receiving NAC prior to cystectomy versus cystectomy alone.

Unfortunately, adoption of cisplatin-based NAC prior to cystectomy was slow $(16,17)$. Investigation of the National Cancer Database showed that from 2004 to 2013, the utilization of NAC in patients undergoing RNU increased from $0.7 \%$ to $2.1 \%$ (18). Potential barriers to utilization were thought to be delayed time to cystectomy, and the possibility that chemotherapy-related complications might render patients ineligible for cystectomy altogether (19). However, in 2000 data was published demonstrating the efficacy of gemcitabine and cisplatin in the treatment of metastatic bladder cancer, with an improved side effect profile over standard MVAC (20). This regimen was subsequently accepted as a more tolerable NAC alternative to MVAC. More recently a dose-dense MVAC regimen was shown in a prospective phase II trial to have similar or improved toxicity profiles compared with standard MVAC in the neoadjuvant setting, with the added benefit of a shorter time to cystectomy (median 9.7 weeks) compared to both standard MVAC and standard gemcitabine/cisplatin dosing (21). The rate of complete pathologic response (38\%; $95 \% \mathrm{CI}, 23 \%$ to $53 \%$ ) in the bladder at cystectomy (pT0), a presumed surrogate for disease specific survival, compared favorably with earlier regimens.

The data from early randomized controlled trials which confirmed a survival advantage associated with NAC, combined with more recent studies identifying more tolerable regimens, has made NAC prior to cystectomy part of the standard of care in muscle invasive bladder cancer. Given the urothelial origins of upper tract cancer, it seems reasonable to expect a similar benefit associated with NAC prior to RNU for UTUC.

\section{Evidence for the efficacy of neoadjuvant chemotherapy in upper tract cancer}

The theoretical goal of NAC in urothelial cancer is the eradication of micro-metastasis, the driver of disease recurrence and cancer-specific mortality after successful extirpative surgery in the clinical N0, M0 setting. Difficulties in measuring this benchmark, have led to the establishment of pathologic downstaging as a surrogate for response to NAC. Grossman et al. demonstrated that downstaging to $<\mathrm{pT} 1$ drives the survival advantage seen with neoadjuvant MVAC in their muscle invasive bladder cancer population (14). In a recent UTUC series, both pathologic complete response and pathologic downstaging by at least one stage (i.e., cT2 to pT1) were associated with improved overall survival (22).

Early multi-institutional data suggested efficacy for NAC in UTUC prior to RNU. In 2009, Margulis et al. published on 1,363 patients undergoing RNU at eleven centers (8). Only $3 \%$ of the combined cohort received NAC prior to RNU. The overall rate of downstaging to pT0 was $<1 \%$ for the cohort, but was $12 \%$ in patients treated with NAC.

Matin et al. first published the M.D. Anderson experience with NAC in UTUC in 2010 (23). Forty-three patients received NAC between 2004 and 2008. These patients had biopsy proven, high-grade UTUC, and the cohort was enriched for high-risk features like sessile tumor architecture. The comparison group was a historical cohort, treated with RNU alone between 1993 and 2003. Patients in the group receiving NAC were significantly more likely to be downstaged at RNU $(\mathrm{P}=0.004)$. The rate of pathologic complete response (pT0) in the NAC cohort was $14 \%$ vs. none in the RNU alone group, and patients with locally advanced disease at RNU ( $\geq \mathrm{pT} 2$, any $\mathrm{N})$ were significantly less common in the NAC treated group ( $46.5 \%$ vs. $65.4 \%, \mathrm{P}=0.043$ ). In a 2014 update of the original manuscript, survival data indicated improved overall and disease specific survival in patients treated with NAC prior 
to RNU compared with a matched cohort undergoing initial RNU (24). The disease specific and overall survival at 5 years in the NAC treated group were $90 \%$ and $80 \%$ respectively, compared to $57.6 \%$ and $57.6 \%$ respectively for the RNU alone cohort.

More recently a retrospective study evaluated the outcomes of 95 patients undergoing RNU for UTUC at two institutions (25). Of the 95 patients undergoing surgery, 61 had renal function that permitted the use of cisplatinbased NAC, 25 of whom received NAC. $80 \%$ of patients receiving NAC had clinical response by imaging, and $80 \%$ had $<$ pT2 disease at surgery $v$ s. only $36 \%$ of the group not receiving NAC. These encouraging outcomes at surgery were reflected in improved progression free survival and overall survival in the NAC-treated cohort. A Japanese study published in 2017 compared two matched cohorts of patients undergoing RNU for locally advanced UTUC, one with NAC and one without NAC (26). Fifty-one matched pairs were selected from 233 patients undergoing RNU between 1995 and 2016. The NAC group demonstrated significantly better 5 -year progression free survival $(60 \%$ vs. $39 \%, \mathrm{P}=0.018)$, cancer specific survival $(71 \%$ vs. $54 \%$, $\mathrm{P}=0.015)$ and overall survival $(65 \%$ vs. $50 \%, \mathrm{P}=0.032)$. NAC was an independent predictor of both progressionfree and cancer-specific survival on multivariate analysis. Almassi et al. reviewed the National Cancer Database for patients undergoing RNU between 2006 and 2014 (27). The outcome of interest in this study was pT0 rate at RNU which was significantly higher in the NAC-treated population $(6.1 \%$ vs. $0.4 \% ; \mathrm{P}<0.001)$, despite the fact that the NAC-treated patients were more likely to have clinically advanced disease (T2-4 in 47\% vs. 29\%; $\mathrm{P}<0.001)$. Receipt of NAC was an independent predictor of pT0 at RNU (OR $19.8,95 \%$ CI, 11.8-33.5).

Most recently Margulis et al. presented the results of the prospective phase II ECOG-ACRIN 8141 trial (28). Thirty-six patients received neoadjuvant therapy in the form of ddMVAC $(\mathrm{CrCl}>50 \mathrm{~mL} / \mathrm{min}, \mathrm{n}=30)$ or gemcitabine/cisplatin $(\mathrm{CrCl} 30-50 \mathrm{~mL} / \mathrm{min}, \mathrm{n}=6)$. While the gemcitabine/cisplatin arm closed to accrual early, the ddMVAC arm demonstrated downstaging to $\leq \mathrm{pT} 1$ in $62 \%$ of patients. Grade 3-4 toxicities were reported in $23 \%$ of the ddMVAC patients, and the regimen was deemed acceptable for further study.

In summary, the existing data supports the efficacy of cisplatin-based NAC in patients with UTUC prior to RNU. However, prospective trials are lacking, making it difficult to advocate in favor of NAC over adjuvant therapy based on oncologic outcomes alone.

\section{Work-up and patient selection for neoadjuvant chemotherapy in upper tract cancer}

The existing data, while limited, suggests oncologic benefit for NAC in UTUC; however, utilization of NAC remains limited. Almassi's group identified 6,174 patients undergoing RNU in the United States between 2006 and 2014 (27). Only $4.2 \%$ received NAC prior to surgery, though utilization of chemotherapy increased from $1.9 \%$ to $7.1 \%$ over the study period. Lack of consistent clinical staging modalities limits clinicians' ability to preoperatively select patients who are at risk of harboring muscle invasive disease at RNU.

Preoperative urine cytology has been shown to predict both $\geq$ pT3 and lymph node involvement at RNU $(29,30)$, but is less consistent as a predictor of $\geq \mathrm{pT} 2$. Messer et al. evaluated 326 patients undergoing RNU and found that cytology was not a sensitive predictor of muscle invasion in UTUC (sensitivity 62\%, PPV 44\%) (31).

Similarly, hydronephrosis has been shown in a large multiinstitutional study, to be an independent predictor of muscle invasive disease at RNU (HR 7.4, 95\% CI, 4.6-11.8), nonorgan confined disease (HR 5.5, 3.4-8.9), and high grade disease (HR 1.6, 1.0-2.6).(32) The association between pre-operative hydronephrosis and advanced disease has not been completely consistent across studies however. A large retrospective Japanese cohort, consisting of 722 patients undergoing RNU was reported in 2015 (30). While hydronephrosis was independently associated with lymphovascular invasion (OR 2.27, 95\% CI, 1.17-4.54), there was no clear association between hydronephrosis and either advanced pathologic stage or high grade disease at RNU.

Ureteroscopic findings of sessile tumor architecture may also predict aggressive disease at the time of definitive resection $(33,34)$. Remzi et al. reviewed the pathology slides of 1363 patients after RNU and categorized gross tumor architecture as papillary (72\%) or sessile (28\%) (34). Sessile tumor architecture was associated with advanced pathologic stage and lymph node involvement, and was an independent predictor of cancer recurrence and cancerspecific mortality. These findings were confirmed by a multiinstitutional study of 754 patients using a similar protocol to evaluate RNU specimens as sessile or papillary (33). About $20 \%$ of the cohort had sessile tumors, of which $95 \%$ were associated with $\geq \mathrm{pT} 2$ and $98 \%$ were associated with high grade disease. On multivariable analysis, macroscopic 
sessile architecture predicted recurrence free survival (HR 1.5, 95\% CI, 1.03-2.1) and cancer-specific survival (HR 1.5, 95\% CI, 1.03-2.2). Ureteroscopic biopsy findings are traditionally of limited utility in predicting pathologic stage. Brown et al. reported that ureteroscopic biopsy grade, not stage, correlated with pathologic stage at RNU (35). High grade biopsies (grade 3 ) correlated with $\geq \mathrm{pT} 2$ : positive predictive value $66 \%$; negative predictive value $72 \%$.

Multiple attempts have been made to develop multivariable models with increased predictive power as a means of preoperatively identifying UTUC patients at high risk of progression after RNU. In 2010, the Upper Tract Urothelial Carcinoma Collaborative Group published a nomogram that included preoperative grade, architecture, and tumor location (36). The model achieved $77 \%$ accuracy in predicting pT3-4 and/or $\mathrm{N}+$ disease at RNU. This was followed in 2012 by an effort from Memorial SloanKettering their cohort of 274 patients (37). The MSKCC model included only local invasion on imaging and high grade tumor on ureteroscopy as independent predictors of nonorgan confined disease on multivariable analysis. The final model had an area under the curve (AUC) of 0.71 for predicting $\geq \mathrm{pT} 2$ and 0.70 for predicting $\mathrm{pT} 3-4$ and/ or $\mathrm{N}+$. More recently in 2019, Margulis et al. published a nomogram based on 245 patients. On multivariable analysis only sessile architecture was an independent predictor of recurrence (HR 2.52; 95\% CI, 1.09-5.86). A nomogram predicting 2 - and 5 -year recurrence free survival was constructed including age, ECOG score, hydronephrosis, architecture, eGFR, clinical stage $\geq \mathrm{T} 3$, and hemoglobin. Patients with greater than three risk factors had 5-year recurrence free survival of $43 \% v s$. those with three or less (78\%). AUC for the model was 0.71 .

Current NCCN recommendations guiding patient selection for NAC prior to RNU suggest that NAC should be "considered for select patients with UTUC, particularly for higher stage and/or grade tumors..." (9). At our institution we refer patients with high grade disease on biopsy, sessile appearing architecture, and/or radiographic findings suggestive of invasion (cT2-4 and/or $\mathrm{cN}+$ ) to medical oncology for a discussion of cisplatin-based NAC.

\section{Evidence for the efficacy of adjuvant chemotherapy in upper tract cancer}

By selecting patients for systemic therapy based on their pathologic findings at RNU, adjuvant chemotherapy programs avoid the dilemma of patient selection based on imperfect pre-operative clinical staging modalities. However, as discussed later, the delivery of chemotherapy in the adjuvant setting may be limited by the patient's health following RNU. Postoperative complications may delay the provision of chemotherapy, and RNU-related decrements in renal function may preclude the use of nephrotoxic chemotherapy altogether.

Initial studies evaluating oncologic outcomes with adjuvant chemotherapy were retrospective in nature, and reported mixed results. Two early multi-institutional studies showed no oncologic advantage to adjuvant systemic therapy after RNU. Hellenthal et al. reported on 542 patients with high risk features at RNU (pT3-4 and/or positive LN), 121 of whom received adjuvant chemotherapy (38). There was no overall or cancer-specific survival advantage associated with adjuvant chemotherapy in this cohort. However, the group receiving adjuvant chemotherapy was enriched with higher risk patients based on pathologic features at surgery (lymph node positivity in adjuvant chemotherapy $v s$. no adjuvant chemotherapy, $43 \%$ vs. $18 \%$ ). Vassilakopoulou et al. evaluated 627 patients who were $\geq \mathrm{pT} 3, \mathrm{~N}+$ or $\mathrm{M}+$, of which 140 received postoperative chemotherapy (39). Adjuvant therapy did not confer better cancer-specific or overall survival. However, only 109 patients received therapy with adjuvant intent for regionally confined disease, the remainder had distant metastasis at RNU and were treated with palliative intent. It is not surprising that neither of these studies demonstrated an oncologic advantage associated with adjuvant therapy, given the enrichment of the adjuvant therapy groups with advanced, and in some cases distantly metastatic, disease.

In 2006 a Korean group published their initial experience with adjuvant chemotherapy in 19 patients who were $\geq \mathrm{p} T 2, \mathrm{~N}$ any, M0 (40). Over a median follow-up period of 30.7 months (range, 4.7 to 98.8 months), $82 \%$ of the non-chemotherapy patients vs. $28 \%$ of the adjuvant chemotherapy patients expired. Adjuvant chemotherapy was associated with overall survival on multivariate analysis. The same group updated their data in 2015, reporting on 139 patients with pT34 or $\mathrm{N}+(41)$. Of the 66 patients who received adjuvant chemotherapy, 60 received cisplatin-based regimens. The authors could show no oncologic advantage associated with adjuvant chemotherapy, but again the group receiving systemic adjuvant therapy was enriched for $\mathrm{N}+$ patients (18\% vs. 4\%). A multi-institutional retrospective study was published by Huang et al. in 2015, comparing outcomes in 60 patients who received adjuvant chemotherapy after RNU vs. 111 who did not (42). Patients were specifically selected 
pT3N0M0, and in this case, adjuvant chemotherapy was associated with both 5 -year cancer-specific $(80.5 \%$ vs. $57.6 \%, \mathrm{P}=0.010)$ and recurrence-free survival $(74.4 \% v s$. $52.9 \%, \mathrm{P}=0.026$ ), as well as a trend toward improved overall survival. On multivariable analysis, adjuvant chemotherapy remained an independent predictor of cancer-specific survival.

More recently, Seisen et al. queried the NCDB for patients undergoing RNU between 2004 and 2012 for pT3-4 and/or pN+ (43). Of 3,253 patients, 762 received adjuvant chemotherapy. Over a median follow-up of almost 50 months, an overall survival advantage emerged for patients getting adjuvant chemotherapy (median (IQR) overall survival for adjuvant chemotherapy $v s$. no adjuvant chemotherapy: 47.4 months (19.9 to 112.4 months) vs. 34.8 months (14.1 to 99 months). This association between overall survival and adjuvant chemotherapy was maintained on multivariable analysis (HR $0.77,95 \%$ CI, 0.68 to 0.88 ; $\mathrm{P}<0.001)$.

The first prospective randomized study of perioperative chemotherapy in UTUC (POUT), was completed recently and presented in February of 2018 (44). The authors accrued 345 patients undergoing RNU, between 2012 and 2017. Patients received either 4 cycles of gemcitabine/ cisplatin within 90 days following surgery, or surveillance. The trial was closed when it met criteria for early termination. The primary endpoint was disease free survival at 2 years which was 0.70 (95\% CI, 0.58 to 0.79$)$ in patients receiving adjuvant therapy and 0.51 (95\% CI, 0.39 to 0.61 ) in the surveillance group. Given the evidence presented above, it seems plausible that adjuvant chemotherapy after RNU, when provided to properly selected patients, may confer some oncologic advantage.

\section{Barriers to adjuvant chemotherapy in UTUC}

Adjuvant therapy after RNU is appealing in comparison to NAC because the ability to base clinical decision making on the pathology specimen avoids the uncertainty of pre-operative staging and potential for over-treatment associated with selecting patients for NAC. However, the act of undergoing RNU may preclude patients from therapy.

Raman et al. reported that while nearly half of their surgical cohort (177/414) had locally advanced disease, only $31 \%$ of locally advanced patients went on to adjuvant therapy (13). Factors preventing the delivery of adjuvant therapy were the $26 \%$ overall complication rate of which one quarter were grade III or IV, and the surgically induced decline in renal function. Receipt of adjuvant chemotherapy was directly related to Clavien grade of complications. $71 \%$ of patients with Clavien grade I or II complications received adjuvant therapy; however, only $17 \%$ of patients with grade III or IV complications eventually received chemotherapy post-RNU ( $\mathrm{P}=0.004)$.

Several groups have evaluated the effect of RNU on eGFR in this patient population. In 2010 Lane et al. published on 336 patients undergoing RNU at the Cleveland Clinic (45). Using eGFR $\geq 60 \mathrm{~mL} / \mathrm{min} / 1.73 \mathrm{~m}^{2}$ calculated by MDRD as a cutoff for chemotherapy eligibility, they demonstrated that only $48 \%$ of their patients were eligible for cisplatin-based therapy prior to RNU. Following RNU only $22 \%$ of patients still qualified for chemotherapy by eGFR criteria. In the same year, Kaag et al. reported on a multi-institutional retrospective effort that evaluated 388 patients (46). eGFR was calculated via the MDRD method prior to, and 3 months after RNU. eGFR cutoffs for chemotherapy eligibility were set at $\geq 60 \mathrm{~mL} / \mathrm{min} / 1.73 \mathrm{~m}^{2}$ and $\geq 45 \mathrm{~mL} / \mathrm{min} / 1.73 \mathrm{~m}^{2}$, with $45 \mathrm{~mL} / \mathrm{min} / 1.73 \mathrm{~m}^{2}$ used as the cutoff for split dose cisplatin at the participating institutions. Prior to RNU $49 \%$ and $80 \%$ of patients were eligible for cisplatin using eGFR cutoffs of $\geq 60 \mathrm{~mL} / \mathrm{min} / 1.73 \mathrm{~m}^{2}$ and $\geq 45 \mathrm{~mL} / \mathrm{min} / 1.73 \mathrm{~m}^{2}$ respectively. After nephrectomy only $19 \%$ and $55 \%$ of patients remained eligible for adjuvant therapy using cutoffs of $\geq 60 \mathrm{~mL} / \mathrm{min} / 1.73 \mathrm{~m}^{2}$ and $\geq 45 \mathrm{~mL} / \mathrm{min} / 1.73 \mathrm{~m}^{2}$ respectively. The eGFR decrement was more pronounced in patients older than 70. An international, multi-institutional study published in 2013 confirmed these findings in 666 patients (47). The average loss of eGFR was $18 \%$ in this study. Using an eGFR cutoff of $\geq 60 \mathrm{~mL} / \mathrm{min} / 1.73 \mathrm{~m}^{2}$, $37 \%$ of patients qualified for chemotherapy prior to RNU, only $16 \%$ qualified after surgery. Using an eGFR cutoff of $\geq 45 \mathrm{~mL} / \mathrm{min} / 1.73 \mathrm{~m}^{2}, 72 \%$ of patients were eligible for chemotherapy before RNU, falling to $52 \%$ after surgery.

Investigators have attempted to predict the degree of renal loss associated with RNU, and in so doing identify those patients for whom the provision of chemotherapy prior to nephrectomy is essential. Body mass index (48), contralateral kidney volume (48), Charlson index (49), and pre-operative hydronephrosis $(50,51)$ have all been shown to be independent predictors of post-RNU renal function. However, age at surgery (49-51) and pre-operative eGFR (48-51) remain the most consistent predictors of post-operative renal function. Hashimoto et al. were able to use age, pre-operatieve eGFR, and pre-operative 
hydronephrosis to develop a model predicting post-RNU renal function (51). The correlation coefficient for the model was 0.75 . Thus, while the adjuvant setting may allow more accurate selection of patients for cisplatin-based chemotherapy based on pathologic criteria, the sequelae of RNU may prevent the administration of nephrotoxic chemotherapy in a fashion that we cannot reliably predict prior to surgery.

\section{Molecular predictors of chemo-response in the perioperative setting}

A significant amount of work has been done evaluating molecular markers of tumor response to cisplatinbased NAC in bladder cancer, which may potentially be extrapolated to predict tumor response in UTUC. Plimack et al. evaluated a panel of DNA repair genes including $A T M, R B$, and FANCC (52). Alterations in one or more of these genes correlated with pathologic response to NAC. $87 \%$ of patients achieving $\leq \mathrm{pT} 1, \mathrm{~N} 0$ after MVAC had alterations in at least one of the three genes $v s$. none in the nonresponsive group. BRCA1 is a known tumor suppressor which identifies damaged DNA for repair. Font et al. demonstrated that low and intermediate levels of $B R C A 1$ correlate with increased NAC response $v s$. high levels of BRCA1 (66\% vs. $22 \%, \mathrm{P}=0.01)$ as judged by final pathologic stage $\leq \mathrm{pT} 1 \mathrm{~N} 0$ (53). ERBB2 codes for a receptor tyrosine kinase that is upregulated in bladder cancer. In one study, ERBB2 missense mutations were found in $24 \%$ of patients achieving pT0 after NAC, compared to $0 / 33$ nonresponders.

Furthermore, gene expression profiling and molecular subtyping in bladder cancer has led to the categorization of tumors into basal, luminal and p53-like subtypes (54). This molecular profiling may be used in bladder cancer to predict response to chemotherapy. For instance, while the basal subtype is associated with aggressive disease manifested as higher rates of metastatic disease at presentation and shorter cancer-specific survival, it has been demonstrated that an immune infiltrated subset of these tumors responds well to NAC. On the other hand, p53-like tumors were consistently resistant to cisplatin-based chemotherapy. These findings may or may not extrapolate to UTUC.

These molecular markers, among others, may add power to current preoperative models predicting pathologic stage at RNU. Further work is needed, specifically in the upper tract domain, to determine the utility of molecular markers as prognostic tools allowing the selection of patients for NAC prior to RNU.

\section{Future directions}

Currently, there is good evidence to support the use of adjuvant chemotherapy in patients with high-risk UTUC at the time of RNU. However, diminished renal function as a result of patient comorbidities combined with nephrectomy remains a very real barrier to the utilization of adjuvant cisplatin-based chemotherapy. At present, existing guidelines recommend consideration of NAC in patients with predictors of advanced disease at RNU, who have sufficient renal function to tolerate cisplatin-based regimens. Careful patient selection is based on the presence of preoperative characteristics, which are imperfect predictors of advanced disease at RNU. An improved understanding of the molecular predictors of advanced disease might allow the development of a preoperative nomogram that could accurately predict disease state at RNU. A review of the $\mathrm{NIH}$ trial listing at clinicaltrials.gov reveals 4 active trials involving neoadjuvant chemotherapy for UTUC. Two trials are evaluating gemcitabine/cisplatin, and two are comparing dose dense MVAC with gemcitabine/cisplatin in one, and gemcitabine/carboplatin in the other. The results from a well-designed clinical trial prospectively evaluating NAC in the UTUC population prior to RNU will clearly be beneficial.

\section{Acknowledgments}

Funding: None.

\section{Footnote}

Provenance and Peer Review: This article was commissioned by the Guest Editors (John J. Knoedler and Jay D. Raman) for the series "Upper-Tract Urothelial Carcinoma: Current State and Future Directions" published in Translational Andrology and Urology. The article was sent for external peer review organized by the Guest Editors and the editorial office.

Conflicts of Interest: The author has completed the ICMJE uniform disclosure form (available at http://dx.doi. org/10.21037/tau.2020.03.48). The series "UpperTract Urothelial Carcinoma: Current State and Future 
Directions" was commissioned by the editorial office without any funding or sponsorship. The author has no other conflicts of interest to declare.

Ethical Statement: The author is accountable for all aspects of the work in ensuring that questions related to the accuracy or integrity of any part of the work are appropriately investigated and resolved.

Open Access Statement: This is an Open Access article distributed in accordance with the Creative Commons Attribution-NonCommercial-NoDerivs 4.0 International License (CC BY-NC-ND 4.0), which permits the noncommercial replication and distribution of the article with the strict proviso that no changes or edits are made and the original work is properly cited (including links to both the formal publication through the relevant DOI and the license). See: https://creativecommons.org/licenses/by-nc-nd/4.0/.

\section{References}

1. Siegel RL, Miller KD, Jemal A. Cancer statistics, 2019. CA Cancer J Clin 2019;69:7-34.

2. Raman JD, Messer J, Sielatycki JA, et al. Incidence and survival of patients with carcinoma of the ureter and renal pelvis in the USA, 1973-2005. BJU Int 2011;107:1059-64.

3. Munoz JJ, Ellison LM. Upper tract urothelial neoplasms: incidence and survival during the last 2 decades. J Urol 2000;164:1523-5.

4. Raman JD, Scherr DS. Management of patients with upper urinary tract transitional cell carcinoma. Nat Clin Pract Urol 2007;4:432-43.

5. Rink M, Sjoberg D, Comploj E, et al. Risk of cancerspecific mortality following recurrence after radical nephroureterectomy. Ann Surg Oncol 2012;19:4337-44.

6. Brown GA, Busby JE, Wood CG, et al.

Nephroureterectomy for treating upper urinary tract transitional cell carcinoma: Time to change the treatment paradigm? BJU Int 2006;98:1176-80.

7. Hall MC, Womack S, Sagalowsky AI, et al. Prognostic factors, recurrence, and survival in transitional cell carcinoma of the upper urinary tract: a 30-year experience in 252 patients. Urology 1998;52:594-601.

8. Margulis V, Shariat SF, Matin SF, et al. Outcomes of radical nephroureterectomy: a series from the Upper Tract Urothelial Carcinoma Collaboration. Cancer 2009;115:1224-33.

9. NCCN. Bladder Cancer. In: NCCN Clinical Practice
Guidelines in Oncology. 2019. 2010.

10. Rouprêt M, Babjuk M, Compérat E, et al. European Association of Urology Guidelines on Upper Urinary Tract Urothelial Carcinoma: 2017 Update. European Urology 2018;73:111-22.

11. Secin FP, Koppie TM, Salamanca JI, et al. Evaluation of regional lymph node dissection in patients with upper urinary tract urothelial cancer. Int J Urol 2007;14:26-32.

12. Hall MC, Womack JS, Roehrborn CG, et al. Advanced transitional cell carcinoma of the upper urinary tract: patterns of failure, survival and impact of postoperative adjuvant radiotherapy. J Urol 1998;160:703-6.

13. Raman JD, Lin YK, Kaag M, et al. High rates of advanced disease, complications, and decline of renal function after radical nephroureterectomy. Urol Oncol 2014;32:47.e9-14.

14. Grossman HB, Natale RB, Tangen CM, et al. Neoadjuvant chemotherapy plus cystectomy compared with cystectomy alone for locally advanced bladder cancer. $\mathrm{N}$ Engl J Med 2003;349:859-66.

15. Advanced Bladder Cancer (ABC) Meta-analysis Collaboration. Neoadjuvant chemotherapy in invasive bladder cancer: update of a systematic review and metaanalysis of individual patient data advanced bladder cancer (ABC) meta-analysis collaboration. Eur Urol 2005;48:2025; discussion 205-6.

16. Fedeli U, Fedewa SA, Ward EM. Treatment of muscle invasive bladder cancer: evidence from the National Cancer Database, 2003 to 2007. J Urol 2011;185:72-8.

17. Donat SM. Integrating perioperative chemotherapy into the treatment of muscle-invasive bladder cancer: strategy versus reality. J Natl Compr Canc Netw 2009;7:40-7.

18. Gin GE, Ruel NH, Kardos SV, et al. Utilization of perioperative systemic chemotherapy in upper tract urothelial carcinoma. Urol Oncol 2017;35:192-200.

19. Bajorin DF, Herr HW. Kuhn's paradigms: are those closest to treating bladder cancer the last to appreciate the paradigm shift? J Clin Oncol 2011;29:2135-7.

20. von der Maase H, Hansen SW, Roberts JT, et al. Gemcitabine and cisplatin versus methotrexate, vinblastine, doxorubicin, and cisplatin in advanced or metastatic bladder cancer: results of a large, randomized, multinational, multicenter, phase III study. J Clin Oncol 2000;18:3068-77.

21. Plimack ER, Hoffman-Censits JH, Viterbo R, et al. Accelerated methotrexate, vinblastine, doxorubicin, and cisplatin is safe, effective, and efficient neoadjuvant treatment for muscle-invasive bladder cancer: results of 
a multicenter phase II study with molecular correlates of response and toxicity. J Clin Oncol 2014;32:1895-901.

22. Martini A, Daza J, Poltiyelova E, et al. Pathological downstaging as a novel endpoint for the development of neoadjuvant chemotherapy for upper tract urothelial carcinoma. BJU Int 2019. [Epub ahead of print].

23. Matin SF, Margulis V, Kamat A, et al. Incidence of downstaging and complete remission after neoadjuvant chemotherapy for high-risk upper tract transitional cell carcinoma. Cancer 2010;116:3127-34.

24. Porten S, Siefker-Radtke AO, Xiao L, et al. Neoadjuvant chemotherapy improves survival of patients with upper tract urothelial carcinoma. Cancer 2014;120:1794-9.

25. Meng X, Chao B, Vijay V, et al. High Response Rates to Neoadjuvant Chemotherapy in High-Grade Upper Tract Urothelial Carcinoma. Urology 2019;129:146-52.

26. Hosogoe S, Hatakeyama S, Kusaka A, et al. Platinumbased Neoadjuvant Chemotherapy Improves Oncological Outcomes in Patients with Locally Advanced Upper Tract Urothelial Carcinoma. Eur Urol Focus 2018;4:946-53.

27. Almassi N, Gao T, Lee B, et al. Impact of Neoadjuvant Chemotherapy on Pathologic Response in Patients With Upper Tract Urothelial Carcinoma Undergoing Extirpative Surgery. Clin Genitourin Cancer 2018;16:e1237-42.

28. Margulis V, Puligandla M, Trabulsi EJ, et al. Phase II Trial of Neoadjuvant Systemic Chemotherapy Followed by Extirpative Surgery for Patients with High Grade Upper Tract Urothelial Carcinoma. J Urol 2020;203:690-8.

29. Brien JC, Shariat SF, Herman MP, et al. Preoperative hydronephrosis, ureteroscopic biopsy grade and urinary cytology can improve prediction of advanced upper tract urothelial carcinoma. J Urol 2010;184:69-73.

30. Sakano S, Inamoto T, Inoue R, et al. Positive voided urine cytology predicts worse pathological findings of nephroureterectomy specimens in patients with upper tract urothelial carcinoma: does selective ureteral cytology have an additional efficacy? Jpn J Clin Oncol 2015;45:968-72.

31. Messer J, Shariat SF, Brien JC, et al. Urinary cytology has a poor performance for predicting invasive or high-grade upper-tract urothelial carcinoma. BJU Int 2011;108:701-5.

32. Messer JC, Terrell JD, Herman MP, et al. Multiinstitutional validation of the ability of preoperative hydronephrosis to predict advanced pathologic tumor stage in upper-tract urothelial carcinoma. Urol Oncol 2013;31:904-8.

33. Fritsche HM, Novara G, Burger M, et al. Macroscopic sessile tumor architecture is a pathologic feature of biologically aggressive upper tract urothelial carcinoma. Urol Oncol 2012;30:666-72.

34. Remzi M, Haitel A, Margulis V, et al. Tumour architecture is an independent predictor of outcomes after nephroureterectomy: a multi-institutional analysis of 1363 patients. BJU Int 2009;103:307-11.

35. Brown GA, Matin SF, Busby JE, et al. Ability of clinical grade to predict final pathologic stage in upper urinary tract transitional cell carcinoma: implications for therapy. Urology 2007;70:252-6.

36. Margulis V, Youssef RF, Karakiewicz PI, et al. Preoperative multivariable prognostic model for prediction of nonorgan confined urothelial carcinoma of the upper urinary tract. J Urol 2010;184:453-8.

37. Favaretto RL, Shariat SF, Savage C, et al. Combining imaging and ureteroscopy variables in a preoperative multivariable model for prediction of muscle-invasive and non-organ confined disease in patients with upper tract urothelial carcinoma. BJU Int 2012;109:77-82.

38. Hellenthal NJ, Shariat SF, Margulis V, et al. Adjuvant chemotherapy for high risk upper tract urothelial carcinoma: results from the Upper Tract Urothelial Carcinoma Collaboration. J Urol 2009;182:900-6.

39. Vassilakopoulou M, de la Motte Rouge T, Colin P, et al. Outcomes after adjuvant chemotherapy in the treatment of high-risk urothelial carcinoma of the upper urinary tract (UUT-UC): results from a large multicenter collaborative study. Cancer 2011;117:5500-8.

40. Kwak C, Lee SE, Jeong IG, et al. Adjuvant systemic chemotherapy in the treatment of patients with invasive transitional cell carcinoma of the upper urinary tract. Urology 2006;68:53-7.

41. Kim HS, Lee JS, Jeong CW, et al. Adjuvant chemotherapy for locally advanced upper tract urothelial carcinoma: updated results of the Seoul National University Hospital experience. Int Braz J Urol 2015;41:1067-79.

42. Huang YC, Chen MF, Shi CS, et al. The Efficacy of Postoperative Adjuvant Chemotherapy for Patients with pT3N0M0 Upper Tract Urothelial Carcinoma. J Urol 2015;194:323-9.

43. Seisen T, Krasnow RE, Bellmunt J, et al. Effectiveness of Adjuvant Chemotherapy After Radical Nephroureterectomy for Locally Advanced and/or Positive Regional Lymph Node Upper Tract Urothelial Carcinoma. J Clin Oncol 2017;35:852-60.

44. Birtle A, Chester J, Jones R, et al. editors. Results of POUT: A phase III randomized trial of perioperative 
chemotherapy versus surveillance in upper tract urothelial cancer (UTUC). ASCO Genitourinary Cancers Symposium; San Francisco: 2018.

45. Lane BR, Smith AK, Larson BT, et al. Chronic kidney disease after nephroureterectomy for upper tract urothelial carcinoma and implications for the administration of perioperative chemotherapy. Cancer 2010;116:2967-73.

46. Kaag MG, O'Malley RL, O'Malley P, et al. Changes in renal function following nephroureterectomy may affect the use of perioperative chemotherapy. Eur Urol 2010;58:581-7.

47. Xylinas E, Rink M, Margulis V, et al. Impact of renal function on eligibility for chemotherapy and survival in patients who have undergone radical nephro-ureterectomy. BJU Int 2013;112:453-61.

48. Song W, Sung HH, Han DH, et al. The effect of contralateral kidney volume on renal function after radical nephroureterectomy: Implications for eligibility for neoadjuvant chemotherapy for upper tract urothelial cancer. Urol Oncol 2017;35:114.e1-114.e7.

49. Kaag M, Trost L, Thompson RH, et al. Preoperative predictors of renal function decline after radical

Cite this article as: Kaag MG. Perioperative chemotherapy in the management of high risk upper tract urothelial cancers. Transl Androl Urol 2020;9(4):1881-1890. doi: 10.21037/ tau.2020.03.48 nephroureterectomy for upper tract urothelial carcinoma. BJU Int 2014;114:674-9.

50. Rodríguez Faba O, Palou J, Breda A, et al. Predictive factors for impaired renal function following nephroureterectomy in upper urinary tract urothelial cell carcinoma. Urol Int 2014;92:169-73.

51. Hashimoto T, Ohno Y, Nakashima J, et al. Prediction of renal function after nephroureterectomy in patients with upper tract urothelial carcinoma. Jpn J Clin Oncol 2015;45:1064-8.

52. Plimack ER, Dunbrack RL, Brennan TA, et al. Defects in DNA Repair Genes Predict Response to Neoadjuvant Cisplatin-based Chemotherapy in Muscle-invasive Bladder Cancer. Eur Urol 2015;68:959-67.

53. Font A, Taron M, Gago JL, et al. BRCA1 mRNA expression and outcome to neoadjuvant cisplatinbased chemotherapy in bladder cancer. Ann Oncol 2011;22:139-44.

54. Choi W, Porten S, Kim S, et al. Identification of distinct basal and luminal subtypes of muscle-invasive bladder cancer with different sensitivities to frontline chemotherapy. Cancer Cell 2014;25:152-65. 